\title{
Dopamine $\beta$-Hydroxylation in Diabetes and Diabetic Autonomic Neuropathy
}

\author{
Robert D. Hoeldtke ${ }^{1}$ and Philip L. Stetson \\ Department of Internal Medicine, Division of Endocrinology and Metabolism and the \\ Metabolism Research Unit, and Upjohn Center for Clinical Pharmacology, \\ University of Michigan, Ann Arbor, Michigan 48109
}

Received October 17, 1980

Diabetic autonomic neuropathy is a heterogenous syndrome with diffuse manifestations affecting many organ systems (1). The alteration in adrenergic physiology is particularly complicated since some patients have low circulating norepinephrine (NE) ${ }^{2}$ levels and a blunted NE response to postural stress, whereas others exhibit high plasma NE and an exaggerated response to the assumption of an upright posture $(2,3)$. This condition has accordingly been classified into hypoadrenergic and hyperadrenergic postural hypotension (3). Little is known about the biochemical basis for these disorders of adrenergic function, but there is pharmacological evidence that in the hypoadrenergic form of this disease, the lesion resides in the postganglionic sympathetic neuron (4). The two most important catecholamine biosynthetic enzymes in sympathetic neurons are tyrosine hydroxylase and dopamine $\beta$-hydroxylase (DBH) and decreased activity of either might lead to decreased NE synthesis $(5,6)$ clinically manifested as postural hypotension. It has been suggested that an abnormality of the latter enzyme is critical since circulating DBH is very low $(<20 \%$ of normal) in some patients with diabetic autonomic neuropathy (7). In idiopathic orthostatic hypotension also circulating $\mathrm{DBH}$ levels are low (8) and these patients have difficulty converting

1 To whom requests for reprints should be sent at: General Clinical Research Center. Temple University Hospital, 3401 N. Broad St., Philadelphia, Pa. 19140.

2 Abbreviations used: NE, norepinephrine; NMN, normetanephrine; DA, dopamine: MTA, methroxytyramine; DHPG, dihydroxyphenylglycol; DHPE, dihydroxyphenylethanol: DHMA, dihydroxymadelic acid; DOPAC, dihydroxyphenylacetic acid; MHPG. 3methoxy-4-hydroxyphenylglycol; MHPE, 3-methoxy-4-hydroxyphenylethanol; VMA, 3methoxy-4-hydroxymandelic acid; HVA, 3-methoxy-4-hydroxyphenylacetic acid. 
intravenously administered $\left[{ }^{14} \mathrm{C}\right]$ dopamine (DA) to $\left[{ }^{14} \mathrm{C}\right] \mathrm{NE}$ (9). The present study was undertaken to determine whether the low serum $\mathrm{DBH}$ reported in patients with diabetic autonomic neuropathy might be indicative of a deficit in the ability of their sympathetic neurons to convert dopamine (DA) to NE. We studied this question with a recently developed tritium release assay, specifically designed to assess DBH activity in sympathetic neurons $(10,11)$.

The tritium-release assay of $\mathrm{DBH}$ is based on the in vitro observation that when $\left[\beta-{ }^{3} \mathrm{H}\right] \mathrm{DA}$ is used as substrate for the hydroxylase, a molecule of THO is released for each molecule of $\left[{ }^{3} \mathrm{H}\right] \mathrm{NE}$ formed (10). The $\left[\beta-{ }^{3} \mathrm{H}\right] \mathrm{DA}$ is an equal mixture of $(\mathrm{S})-\left[\beta-{ }^{3} \mathrm{H}\right] \mathrm{DA}$, whose hydroxylation leads to the formation of $\left[{ }^{3} \mathrm{H}\right] \mathrm{NE}$, and $(\mathrm{R})-\left[\beta-{ }^{3} \mathrm{H}\right] \mathrm{DA}$ whose hydroxylation leads to the formation of THO (Scheme 1).

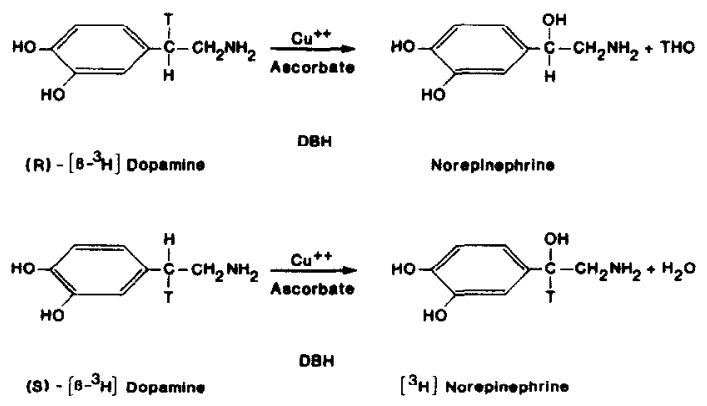

Since half of the labeled product is in the form of THO and half in the form of $\left[{ }^{3} \mathrm{H}\right] \mathrm{NE}$ and its metabolites, it is possible to assay these two indices of dopamine $\beta$-hydroxylation simultaneously. The measurement of THO is of interest since this product of the enzymatic reaction (unlike $\left[{ }^{3} \mathrm{H}\right] \mathrm{NE}$ ) undergoes neither subsequent metabolism nor reuptake into the nerve terminal.

\section{MATERIALS AND METHODS}

\section{Experimental Protocol}

The 19 participants in this study included seven healthy subjects, six diabetics without autonomic neuropathy, and six diabetics with autonomic neuropathy. The two groups of diabetics were well matched for age and sex; the healthy subjects were slightly older (Table 1). All of the diabetics were on insulin therapy; two-thirds of the diabetics in each group were insulin dependent. The mean FBS for the autonomic neuropathy patients was $205 \pm 33$ (SEM); the mean FBS for the diabetics without neuropathy was $156 \pm 40$. None of the patients had renal insufficiency (serum creatinine greater than $1.2 \mathrm{mg} / 100 \mathrm{ml}$ ) or hypertension (diastolic BP greater than $90 \mathrm{~mm} \mathrm{Hg}$ ). Two of the patients with autonomic 
TABLE 1

Characterization of the Healthy Controls and Diabetic Patients Who Participated IN THE In Vivo DBH ASSAY

\begin{tabular}{llll} 
& $\begin{array}{c}\text { Normal } \\
\text { subjects }\end{array}$ & $\begin{array}{c}\text { Diabetics without } \\
\text { autonomic neuropathy }\end{array}$ & $\begin{array}{c}\text { Diabetics with } \\
\text { autonomic neuropathy }\end{array}$ \\
\hline Number of participants & 7 & 6 & 6 \\
Sex & 3 males & 2 males & 2 males \\
& 4 females & 4 females & 4 females \\
Age range (years) & $32-60$ & $28-63$ & $25-63$ \\
$\quad$ Mean & 44 & 40 & 37 \\
Body weight range $(\mathrm{kg})$ & $51.1-109$ & $51.0-78$ & $47.3-81$ \\
$\quad$ & 77 & 64 & 63 \\
\hline
\end{tabular}

neuropathy had nonproliferative retinopathy. None of the diabetics without autonomic neuropathy had clinically manifest complications. The autonomic neuropathy patients had a drop in systolic blood pressure of at least $20 \mathrm{~mm} \mathrm{Hg} 15 \mathrm{sec}$ after shifting from the supine to the upright posure. In addition, these patients had a cardiac beat-to-beat variation on deep breathing of less than 12 (12) and/or a post-valsalva bradycardia tachycardia $\mathbf{R}-\mathbf{R}$ interval ratio of less than 1.15 (13).

$\left[\beta-{ }^{3} \mathrm{H}\right] \mathrm{DA}$ (sp act $20 \mathrm{Ci} / \mathrm{mmole}$, New England Nuclear, NET-131) was passed through a $0.2 \mu$ Millipore filter to protect against bacterial contamination. The isotope $(500 \mu \mathrm{C} / 70 \mathrm{~kg})$ was infused slowly over $2 \mathrm{~min}$ into an antecubital vein to patients at the Clinical Research Center of the University of Michigan. Urine was collected for $\left[{ }^{3} \mathrm{H}\right]$ catecholamine metabolite determination for the first $6 \mathrm{hr}$ after isotope administration in bottles containing $10 \mathrm{ml}$ of $6 \mathrm{~N} \mathrm{HCl}$. The patients remained upright and ambulatory during the urine collection period, though they were allowed to sit down for lunch and a 5-min rest period each hour was permitted. The diabetics were maintained on their usual diet and insulin regimen on the day of the experiment.

Four of the control patients participated in the in vivo assay twice, once with and once without disulfiram pre-reatment $(5.5 \mathrm{mg} / \mathrm{kg} /$ day orally for 4 days). The $\left[\beta-{ }^{3} \mathrm{H}\right] \mathrm{DA}$ was given $90 \mathrm{~min}$ after the last dose of disulfiram. There was at least a 5 -week interval between the control period and the disulfiram treatment period.

This study was approved by the University of Michigan Human Use Committee. Informed consent was obtained from all subjects prior to their participation.

\section{Assay of Total Body THO}

THO was lypohilized from whole blood and trapped according to the method of Stansell and Mojica (14). Total body THO was calculated (on 
the assumption that intravascular THO equals extravascular $\mathrm{THO}$ ) as described previously (11).

\section{Assay of $\left[{ }^{3} \mathrm{H}\right]$ Catecholamines and $\left[{ }^{3} \mathrm{H}\right]$ Catecholamine Metabolites}

\section{Endogenous to NE and HVA}

Urinary $\left[{ }^{3} \mathrm{H}\right]$ catecholamines were extracted and fractionated by ion exchange (Dowex 50W-X4) and alumina chromatography (15). Final separations were performed with high-pressure liquid chromatography (HPLC) (16). Urinary HVA was purified by HPLC (16) and then assayed fluorometrically (17). Free urinary NE was purified by column chromatography (15) and then assayed fluorometrically (18).

\section{RESULTS}

DBH activity of the diabetic autonomic neuropathy patients did not differ significantly from that of the diabetics without neuropathy or the healthy controls (Fig. 1). There were no significant differences in $\left[{ }^{3} \mathrm{H}\right] \mathrm{NE}$ metabolite excretion among these groups of subjects (Table 2) though the ratio of total $\left[{ }^{3} \mathrm{H}\right] \mathrm{NE}$ metabolites to total $\left[{ }^{3} \mathrm{H}\right] \mathrm{DA}$ metabolites was slightly decreased in some of the autonomic neuropathy patients (Fig. 2). As previously reported (11) there was a positive correlation between

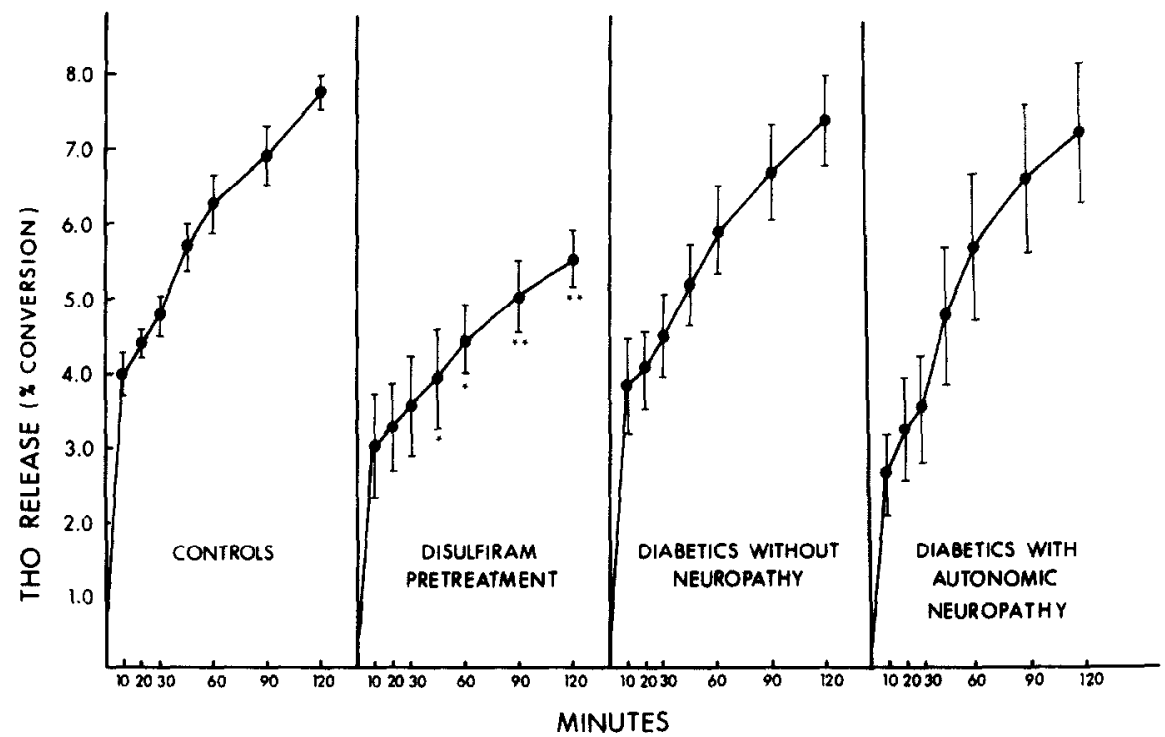

FIG. 1. In vivo DBH Assay in Control Subjects $(N=7)$ following disulfiram treatment $(N=4)$, and in diabetic patients with $(N=6)$ and without $(N=6)$ autonomic neuropathy. The data indicate mean total body THO \pm SEM. expressed as percentage of administered isotope. Statistical analysis was performed as described under Results. Asterisks as defined in Table 2. 


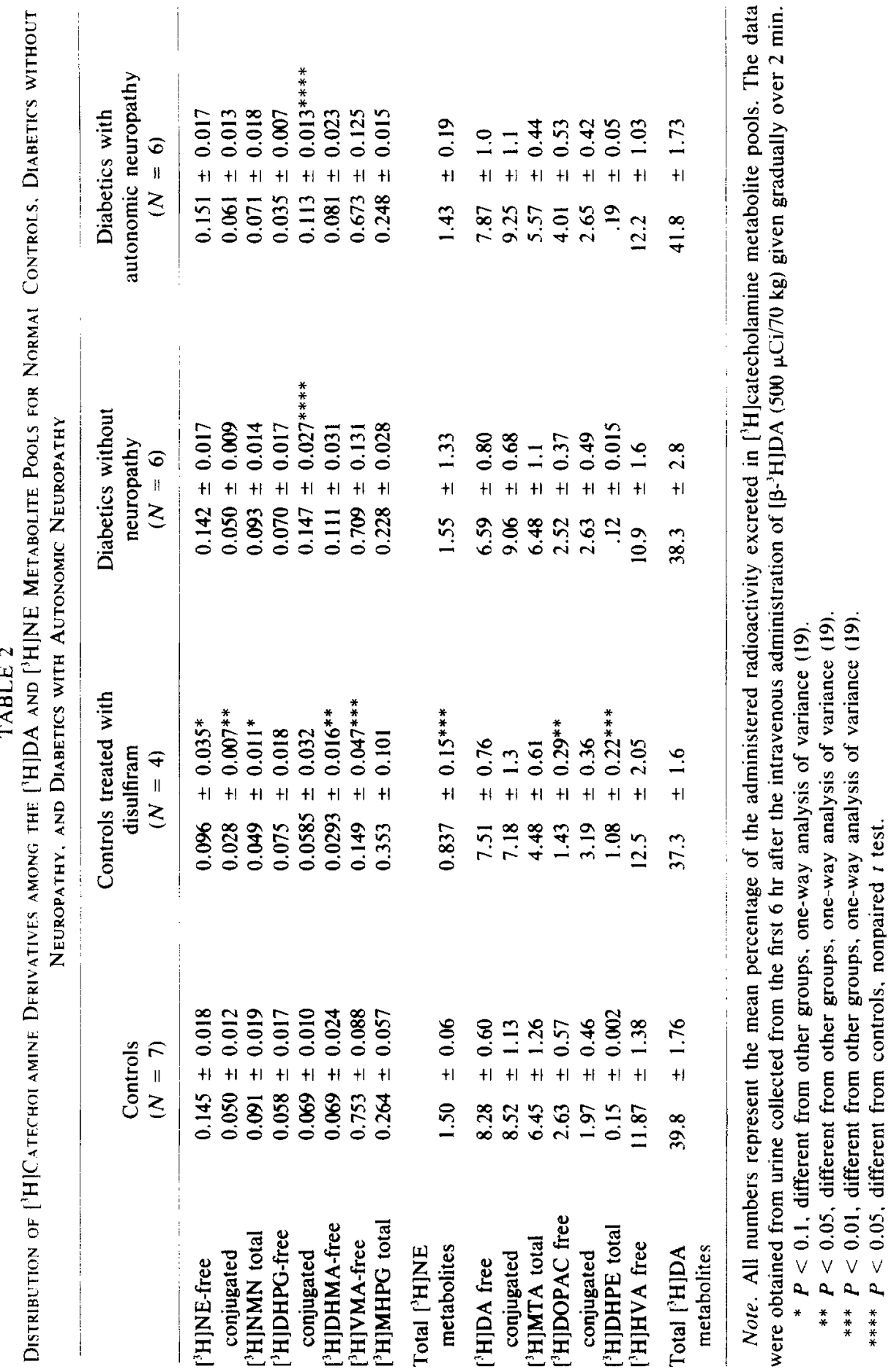




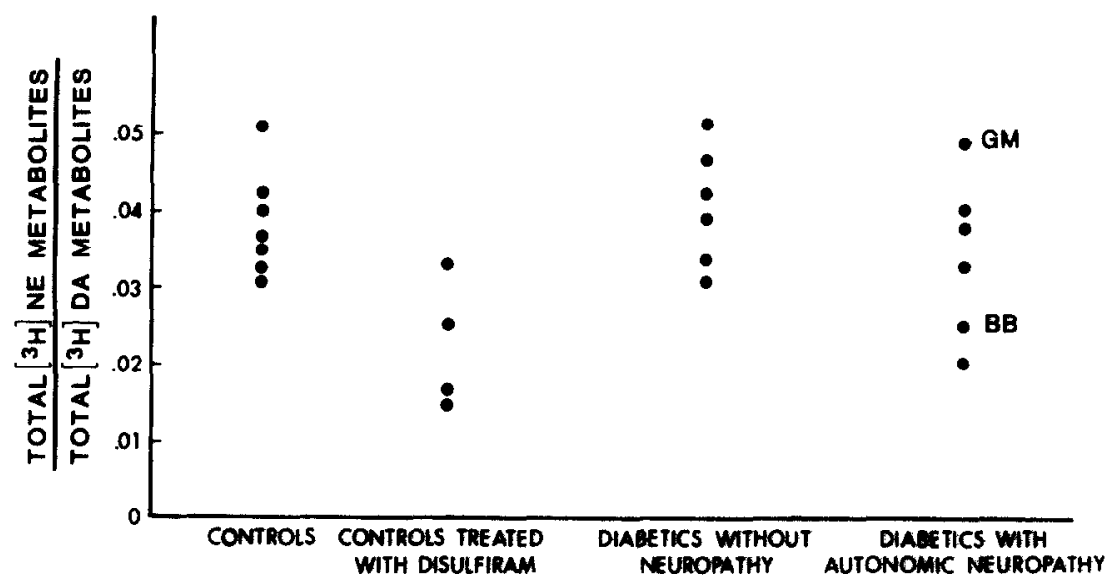

FIG. 2. $\left[{ }^{3} \mathrm{H}\right] \mathrm{NE}$ metabolite excretion in control patients and diabetics, with and without autonomic neuropathy. Each point represents the ratio of total $\left[{ }^{3} \mathrm{H}\right] \mathrm{NE}$ metabolites/total $\left[{ }^{3} \mathrm{H}\right] \mathrm{DA}$ metabolites for a single patient. One-way analysis of variance revealed that this ratio was significantly decreased $(P<0.005)$ in the disulfiram-treated patients specifically compared with the other three groups of patients (19).

THO release and $\left[{ }^{3} \mathrm{H}\right] \mathrm{NE}$ metabolite excretion $(r=0.68, P<0.01$, Fig. $3)$. In order to assess the effect of disulfiram on these two indices of dopamine $\beta$-hydroxylation, a one way analysis of variance was performed and then the disulfiram pretreatment group was specifically compared with the other three groups (19). By this criteria, disulfiram treatment significantly decreased THO release (Fig. 1), the excretion of many of the individual $\left[{ }^{3} \mathrm{H}\right] \mathrm{NE}$ metabolites (Table 2), and total $\left[{ }^{3} \mathrm{H}\right] \mathrm{NE}$ metabolite excretion (Fig. 2). Disulfiram also inhibited aldehyde dehydrogenase as evidenced by the marked increase in the excretion of the minor neutral DA metabolite $\left[{ }^{3} \mathrm{H}\right]$ dihydroxyphenylethanol (DHPE) and the modest decrease in free $\left[{ }^{3} \mathrm{H}\right]$ dihydroxyphenylacetic acid (DOPAC) excretion. ${ }^{3}$

The free NE excretion of the autonomic neuropathy patients was quite variable, and only two of these patients (BB and $\mathrm{GM}$ ) excreted low levels of free NE (Fig. 4). These patients did not exhibit decreased THO release from $\left[\beta-{ }^{3} \mathrm{H}\right] \mathrm{DA}$ and, in fact, one of them (GM) had increased THO release. GM also had low HVA excretion (Fig. 5). There was a negative correlation between HVA excretion and THO release $(r=-.50, P<$ 0.05, Fig. 5).

\section{DISCUSSION}

Our data indicate that most patients with diabetic autonomic neuropathy hydroxylate dopamine normally. This condition is thus distinct

${ }^{3}$ The disulfiram effect on aldehyde dehydrogenase should not affect tritium release and interfere with the in vivo DBH assay. This point is discussed in detail elsewhere (11). 


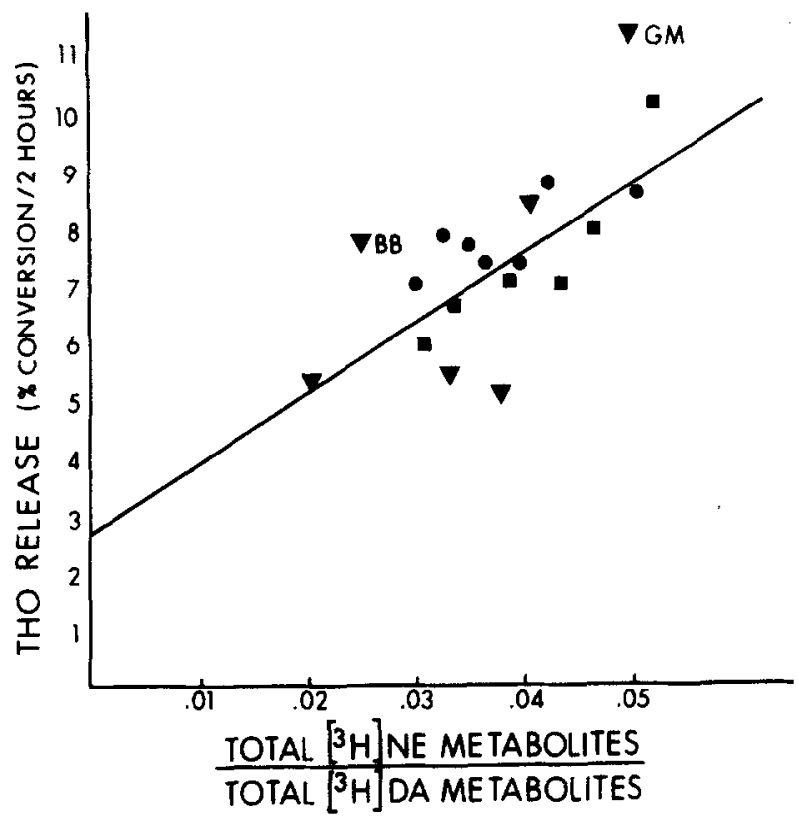

FIG. 3. THO release and $\left[{ }^{3} \mathrm{H}\right] \mathrm{NE}$ metabolite excretion in controls $(O)$ diabetes $(\square)$ and diabetic autonomic neuropathy $(\nabla)$. A highly significant correlation $(r=0.68, P<0.01)$ was observed between THO release and $\left.{ }^{3} \mathrm{H}\right] \mathrm{NE}$ metabolite excretion. The regression equation for the pooled data is $Y=126 \mathrm{X}+2.69$.

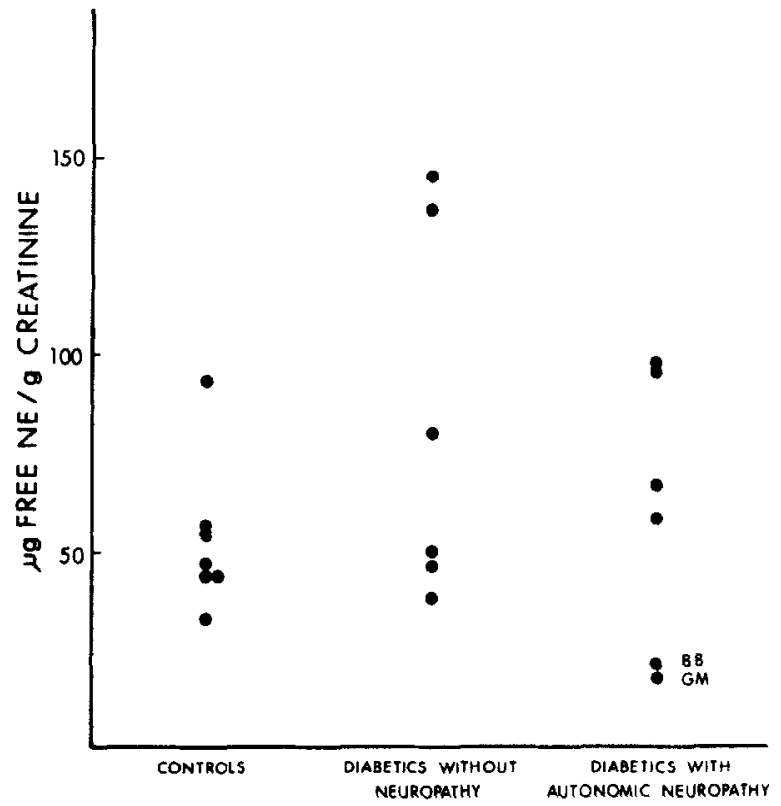

Fig. 4. Free NE excretion in control subjects and diabetics, with and without autonomic neuropathy. There was no significant difference in the free NE excretion of these three groups of patients. 


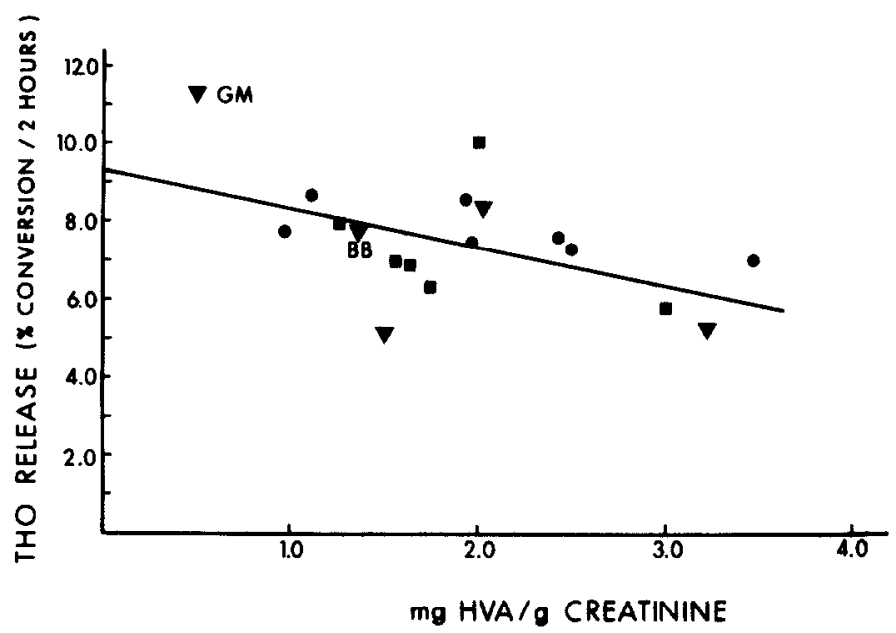

Fig. 5. Negative correlation between THO release from $\left[\beta-{ }^{3} \mathrm{H}\right] \mathrm{DA}$ and endogenous urinary HVA. Symbols as defined in Fig. 3. The regression equation for the pooled data is $Y=-.99 \mathrm{X}+9.43, r=-0.501, P<0.05$ ).

from idiopathic orthostatic hypotension where a deficit in dopamine $\beta$ hydroxylation has been described (9). Though some of the diabetics with autonomic neuropathy may have a mild impairment of DBH activity as evidenced by their slightly decreased $\left[{ }^{3} \mathrm{H}\right] \mathrm{NE}$ metabolite excretion following intravenous $\left[\beta-{ }^{3} \mathrm{H}\right] \mathrm{DA}$ (Fig. 2), this deficit probably could not account for their postural hypotension. This is because some of the disulfiram-treated control subjects exhibited a greater decrease in $\left[{ }^{3} \mathrm{H}\right] \mathrm{NE}$ metabolite excretion than did the neuropathy patients but had little or no change in their blood pressure as they shifted from a supine to an upright posture.

Since we did not measure circulating NE and its response to postural stress, we cannot strictly categorize our patients into hypoadrenergic and hyperadrenergic autonomic neuropathy as defined previously (3). Urine was collected with the patients upright, however, and highly variable urinary NE levels were noted in the autonomic neuropathy group simulating the variability previously observed in plasma NE (3). Since the assumption of the upright posture has a similar effect on plasma NE and urinary NE (20), it is reasonable to assume that the two individuals in the present study (GM and BB) with low urinary NE had hypoadrenergic autonomic neuropathy. Neither of these patients had decreased DBH activity (as assessed from THO release from $\left[\beta-{ }^{3} \mathrm{H}\right] \mathrm{DA}$ ) but of special interest is the fact that one patient (GM) had increased THO release (Figs. 3 and 5). Though the high THO release may represent enhanced DBH activity in his sympathetic neurons, this interpretation 
of the data does not explain the low urinary NE. It thus seems more likely that the high THO release is the result of depletion of the pool of intraneuronal DA resulting in low endogenous substrate competing with $\left[\beta-{ }^{3} \mathrm{H}\right] \mathrm{DA}$ for $\mathrm{DBH}$. In support of this conclusion is the fact that GM's urinary HVA was low (Fig. 5). It is unlikely that a deficit in NE release is the cause of his low urinary NE since $\left[{ }^{3} \mathrm{H}\right] \mathrm{NE}$ metabolite excretion was not decreased (Fig. 2).

This negative correlation between THO release and HVA excretion (Fig. 5) suggests that alterations in the sympathetic neuronal DA pool may affect the in vivo essay of DBH. Though there are no available methods for resolving this issue in humans by determining the specific activity of the intraneuronal DA pool, the tritium release principle can nevertheless be used to clinically estimate the activity of catecholamine biosynthetic enzymes in disease states associated with low NE levels in blood and urine. This is possible because there are only two potentially regulatory enzymes involved in NE biosynthesis, tyrosine hydroxylase and DBH $(5,6)$. Thus, low NE levels coupled with low THO release from $\left[\beta-{ }^{3} \mathrm{H}\right] \mathrm{DA}$ suggest a deficit in dopamine $\beta$-hydroxylation, as observed in subjects treated with disulfiram (11). By contrast when low NE levels are coupled with high THO release, reasoning by exclusion suggests an abnormality in the other potentially regulatory NE biosynthetic enzyme, tyrosine hydroxylase. ${ }^{4}$ Moreover, decreased activity of the latter enzyme would be expected to lead to decreased endogenous DA competing with $\left[\beta-{ }^{3} \mathrm{H}\right] \mathrm{DA}$ for $\mathrm{DBH}$, the result being high THO release. The presently described patient (GM) with low urinary NE, low urinary HVA, and high THO release exemplifies this pattern of results. Further studies, however, will be needed to establish the validity of this interpretation of high rates of THO release from $\left[\beta-{ }^{3} \mathrm{H}\right] \mathrm{DA}$.

\section{SUMMARY}

Dopamine $\beta$-hydroxylase (DBH) has been studied in healthy subjects and diabetic patients, some of whom had autonomic neuropathy. DBH was assayed by a recently described in vivo tritium release method $(10,11)$. Dopamine specifically labeled in the $\beta$ position, $\left[\beta-{ }^{3} \mathrm{H}\right] \mathrm{DA}$, was given intravenously, and the time course of tritiated water (THO) release into the total body water was measure as an index of DBH activity in sympathetic neurons. We simultaneously assayed $\left[{ }^{3} \mathrm{H}\right]$ norepinephrine metabolite excretion.

The DBH activity of patients with autonomic neuropathy as a group did not differ significantly from that of diabetics without neuropathy or

\footnotetext{
4 The third enzyme in the pathway, L. aromatic amino acid decarboxylase. is probably never rate limiting with respect to NE biosynthesis $(5,6)$.
} 
healthy controls. Our data do not support the suggestion (7) that a deficit in DBH activity is responsible for sympathetic neuronal dysfunction in diabetic autonomic neuropathy. This syndrome thus differs from idiopathic orthostatic hypotension in which condition there is an impairment in the ability of sympathetic neurons to hydroxylate dopamine (9).

\section{ACKNOWLEDGMENTS}

Supported in part by Michigan Diabetes Research and Training Center Grant 5-P60-AM20572, University of Michigan Clinical Research Center Grant 5 MOIRR-42, and Division of Research Resources of the National Institute of Health and USPHS Grants AM00888 and T32 AM07245.

\section{REFERENCES}

1. Duchen, L. W., Anjorin, A., Watkins, P. J., and Mackay, J. D., Ann. Intern. Med. 92, 301 (1980).

2. Christensen, N. J., J. Clin. Invest. 51, 779 (1972).

3. Cryer, P. E., Silverberg, A. B., Santiago, J. V., and Shah, S. D., Amer. J. Med. 64, 407 (1978).

4. Leveston, S. A., Shah, S. D., and Cryer, P. E., J. Clin. Invest. 64, 374 (1979).

5. Thoenen, H., Ketter, R., Burkard, W., and Saner, A., Naunyn-Schiedeberg's Arch. Pharmakol. 270, 146 (1971).

6. Black, I. B., Hendry, I., and Iversen, L. L., Nature (London) 231, 28 (1971).

7. Noth, R. H., and Mulrow, P. J., Circ. Res. 38, 2 (1976).

8. Ziegler, M. G., Lake, R. C., and Kopin, I. J., N. Engl. J. Med. 296, 293 (1977).

9. Goodall, McC., Harlan, W. R., and Alton, H., Circulation 38, 592 (1968).

10. Hoeldtke, R. D., and Kaufman, S., Biochem. Pharmacol. 27, 2499 (1978).

11. Hoeldtke, R. D. and Stetson, P. L., J. Clin. Endo. Metab. 51, 810 (1980).

12. Watkins, P. J., and Mackay, J. D., Ann. Intern. Med. 92, 304 (1980).

13. Ewing, D. J., Burt, A. A., Campbell, I. W., and Clarke, B. F., Lancet 2, 1354 (1973).

14. Stansell. M. J., and Mojica, L., Clin. Chem. 14, 1112 (1968).

15. Taylor, K. M., and Laverty, R., J. Neurochem. 16, 1361 (1969).

16. Hoeldtke, R. D., and Stetson, P. L., Anal. Biochem. 105, 207 (1980).

17. Andes, N. E., Roos, B. E., and Werdinius, B., Life Sci. 7, 488 (1963).

18. von Euler, U. S., and Lishajko, F., Acta Physiol. Scand. 45, 122 (1959).

19. Snedecor, G. W., and Cochran, W. G., "Statistical Methods," p. 268. Iowa State Univ. Press, Ames. 1964.

20. Henry D. P., Dentino, M., Gibbs, P. S., and Weinberger, M., J. Lab. Clin. Med. 94, 429 (1979). 